\title{
Imaging Drugs with and without Clinical Analgesic Efficacy
}

\author{
Jaymin Upadhyay ${ }^{1,2}$, Julie Anderson ${ }^{1,2}$, Adam J Schwarz ${ }^{1,3}$, Alexandre Coimbra ${ }^{1,4}$, Richard Baumgartner ${ }^{1,4}$, \\ G Pendse ${ }^{1,2}$, Edward George ${ }^{1,2}$, Lauren Nutile ${ }^{1,2}$, Diana Wallin ${ }^{1,2}$, James Bishop ${ }^{1,2}$, Saujanya Neni ${ }^{1,5}$, \\ Gary Maier $^{1,5}$, Smriti lyengar ${ }^{1,3}$, Jeffery L Evelhoch ${ }^{1,4}$, David Bleakman ${ }^{1,3}$, Richard Hargreaves ${ }^{1,4}$, \\ Lino Becerra ${ }^{1,2,6}$ and David Borsook*,1,2,6 \\ I'Imaging Consortium for Drug Development; ${ }^{2}$ P.A.I.N. Group, Brain Imaging Center, McLean Hospital, Belmont, MA, USA; ${ }^{3}$ Lilly Research \\ Laboratories, Eli Lilly and Company, Indianapolis, IN, USA; ${ }^{4}$ Merck Research Laboratories, West Point, PA, USA; ${ }^{5}$ Sunovion Inc., Marlborough, MA, \\ USA; ${ }^{6}$ Athinoula A Martinos Center for Biomedical Imaging, Massachusetts General Hospital, Harvard Medical School, Charlestown, MA, USA
}

\begin{abstract}
The behavioral response to pain is driven by sensory and affective components, each of which is mediated by the CNS. Subjective pain ratings are used as readouts when appraising potential analgesics; however, pain ratings alone cannot enable a characterization of CNS pain circuitry during pain processing or how this circuitry is modulated pharmacologically. Having a more objective readout of potential analgesic effects may allow improved understanding and detection of pharmacological efficacy for pain. The pharmacological/functional magnetic resonance imaging (phMRI/fMRI) methodology can be used to objectively evaluate drug action on the CNS. In this context, we aimed to evaluate two drugs that had been developed as analgesics: one that is efficacious for pain (buprenorphine (BUP)) and one that failed as an analgesic in clinical trials aprepitant (APREP). Using phMRI, we observed that activation induced solely by BUP was present in regions with $\mu$-opioid receptors, whereas APREP-induced activation was seen in regions expressing NK, receptors. However, significant pharmacological modulation of functional connectivity in pain-processing pathways was only observed following BUP administration. By implementing an evoked pain $\mathrm{FMRI}$ paradigm, these drugs could also be differentiated by comparing the respective $\mathrm{PMRI}$ signals in CNS circuits mediating sensory and affective components of pain. We report a correlation of functional connectivity and evoked pain $\mathrm{fMRI}$ measures with pain ratings as well as peak drug concentration. This investigation demonstrates how CNS-acting drugs can be compared, and how the phMRI/fMRI methodology may be used with conventional measures to better evaluate candidate analgesics in small subject cohorts.

Neuropsychopharmacology (20II) 36, 2659-2673; doi:I0.1038/npp.20II.156; published online 17 August 20I I
\end{abstract}

Keywords: phMRI; evoked $\mathrm{PMRI}$; analgesia; buprenorphine; fosaprepitant

\section{INTRODUCTION}

The identification of effective drugs for pain treatment has proven to be extremely difficult (Hewitt et al, 2009; Katz et al, 2008). It is often the case that pharmacological candidate compounds are efficacious in preclinical pain studies and are progressed to large-scale clinical trials, yet do not show clinical efficacy for pain treatment. Prior to clinical trials involving patients, early clinical evaluation of potential analgesic drugs includes safety and tolerability studies, and pharmacokinetic evaluation in healthy subjects, ideally an assessment of target engagement (eg, receptor occupancy through positron emission tomography (PET)

\footnotetext{
*Correspondence: Dr D Borsook, P.A.I.N. Group, Brain Imaging Center, McLean Hospital, Harvard Medical School, I 5 Mill St Belmont, MA 02478, USA, Tel: + I 617855 3772, Fax: + | 617855 269|, E-mail: dborsook@partners.org

Received 26 February 201।; revised I4 June 201 I; accepted I5 June 2011
}

when a suitable radioactive ligand is available), and possibly an exploration of pharmacodynamic effects in experimental pain models. In early experimental pain models and larger scale clinical pain investigations, subjective pain ratings have been typically used as primary behavioral endpoints. Although measurements such as pain rating are critical in evaluating potential analgesics, pain ratings are intrinsically highly variable measures (Lin et al, 2011; Nicholson, 1978; Steingrimsdottir et al, 2004; Victor et al, 2008) and do not facilitate a comprehension of how or whether the CNS pain circuitry (ie, limbic, mesolimbic, or sensorimotor circuits) are pharmacologically modulated. From pain ratings alone, it is not possible to determine whether the mechanisms of action or targets that were linked to producing analgesia in preclinical studies were successfully engaged in the target clinical population. Knowledge of the pharmacological impact on CNS pain systems may facilitate a better understanding between clinically efficacious and nonefficacious drug mechanisms, and doses for pain treatment. Thus, methodology and pharmacodynamic biomarkers that 
would enable an objective evaluation of drug action on CNS pain systems may be highly valuable.

Previous pharmacological magnetic resonance imaging (phMRI) studies have assessed the feasibility of this technique as a means to define regions of increased activity in the human brain solely owing to drug administration (Becerra et al, 2006; Borras et al, 2004; Leppa et al, 2006). Moreover, blood oxygenation level-dependent (BOLD) functional MRI (fMRI) is a method that can be used to probe the CNS circuitry during a painful stimulus and also in the presence of a pharmacological compound (Iannetti et al, 2005; Wise et al, 2002). Whereas phMRI is in reference to measuring the effects of pharmacological compounds on the resting BOLD signal, fMRI refers to measuring the BOLD signal in response to an evoked stimulation subsequent to drug or placebo administration. Both MRbased methods can be implemented to objectively characterize the neuroanatomical localization of an analgesic effect for a specific pharmacological compound, and used in conjunction with standard clinical methods (ie, subjective pain ratings). This approach may be used for identifying clinically ineffective and effective pharmacology for pain treatment.

Here, drug action on the human CNS pain circuitry was evaluated by using the phMRI and fMRI methodology for two drugs previously appraised as analgesics: a partial $\mu$-opioid agonist and $\kappa$-opioid antagonist, buprenorphine (BUP) (Lutfy and Cowan, 2004), and a neurokinin-1 ( $\left.\mathrm{NK}_{1}\right)$ antagonist, fosaprepitant (Once fosaprepitant (phosphoprodrug) is intravenously administered, it is metabolized into aprepitant (APREP) (also orally active). Following this metabolism, APREP binds to $\mathrm{NK}_{1}$ receptors.) (Patel and Lindley, 2003). BUP has FDA approval as an analgesic (Budd, 1981; Christoph et al, 2005; Cowan et al, 1977; Jasinski et al, 1978; Kogel et al, 2005), however, multiple clinical trials of $\mathrm{NK}_{1}$ antagonists as analgesics have failed (Goldstein et al, 1997, 2001; Hill, 2000). Specifically, APREP failed to demonstrate significant analgesic effects in phaseII clinical trials. BUP and APREP are also ideal comparators given the substantial overlap in the anatomical distribution of their primary target receptors in the brain, whereas their target receptor type and associated cellular mechanisms of action differ. We sought to differentiate the central neurobiological effects of these two compounds with a view to understanding their differential efficacy in pain treatment.

The objectives of the present study were four-fold: (1) To characterize and compare the phMRI infusion responses between BUP and APREP. As a result of the overlap in $\mu$-opioid (Greenwald et al, 2003; Zubieta et al, 2000) and $\mathrm{NK}_{1}$ (Bergstrom et al, 2004; Hietala et al, 2005) receptor distribution, we hypothesized an overlap in phMRI infusion responses between BUP and APREP. (2) To assess whether functional connectivity assessment can quantify how functional interactions in the CNS pain circuitry are altered or preserved for the two pharmacological compounds. We hypothesized that either BUP or APREP could alter functional connectivity in the CNS circuitry involved in the sensory, motivational, and emotional aspects of pain processing in a manner that may provide insights for clinical effects (Bingel et al, 2004; Maihofner et al, 2007; Singer et al, 2004). (3) To measure BOLD fMRI and pain ratings during noxious heat stimulation, and determine whether and how the central effects of BUP and APREP could be differentiated from neurological and behavioral perspective. (4) To define whether and how phMRI/fMRIbased measures relate to other endpoints such as drug concentrations in plasma and subjective pain ratings.

\section{MATERIALS AND METHODS}

Twenty-four healthy, male, right-handed subjects were included in this study (age: $28.0 \pm 2.5$ years old (mean \pm SEM)). Intravenous administration of $0.2 \mathrm{mg} / 70 \mathrm{~kg}$ BUP (Reckitt Benckiser Pharmaceuticals) and $96 \mathrm{mg} / 70 \mathrm{~kg}$ APREP (Merck) was investigated. The present study included a distinct 12-subject cohort for each drug investigated. In each study cohort, subjects underwent two scanning sessions on a Siemens $3 \mathrm{~T}$ Trio machine that were $\sim 2$ weeks apart, where either placebo (saline solution) or BUP/ APREP (drug plus saline solution) was administered intravenously in each scanning session. A randomized, double-blinded, crossover design was implemented. The subjects in the BUP cohort were previously used to develop the general linear model (GLM) analysis for phMRI infusion data (Pendse et al, 2010b). One subject in the BUP cohort consumed anti-histamines on an as needed basis; however, all other subjects were on no medications. Subjects reported no history of pain or drug abuse. Each subject gave informed consent prior to entering the study. The McLean Hospital Institutional Review Board approved this investigation.

\section{Subject Preparation}

Specific study protocols were implemented for subject preparation; intravenous administration of BUP, APREP, or saline; drug concentration measurements; Quantitative Sensory Testing (QST); imaging data acquisition; and image preprocessing. Details on these procedures have been given under Supplementary Materials and Methods.

\section{Evoked Pain (Heat) Stimulation}

Heat fMRI data were collected $\sim 35$ and $\sim 25$ min after completion of BUP and APREP administration, respectively. During the fMRI scan, noxious heat stimuli were applied to the dorsum of the subjects' left foot. During the $5 \mathrm{~min} 10 \mathrm{~s}$ fMRI scan, seven heat stimulations were delivered in a 25/15-s off/on cycle. VAS pain ratings were collected continuously throughout the fMRI scan. The mean \pm SEM target temperature used during the evoked pain fMRI scan for the BUP and APREP cohorts was $46.88 \pm 0.66^{\circ} \mathrm{C}$ and $47.80 \pm 0.44^{\circ} \mathrm{C}$, respectively.

\section{Image Analysis}

Single-subject GLM (univariate) analysis of phMRI data. All imaging analysis was performed by using the FMRIB Software Library (FSL) (www.fmrib.ox.ac.uk/fsl). To extract neuronal structures possessing a phMRI infusion response to $0.2 \mathrm{mg} / 70 \mathrm{~kg}$ BUP or $96 \mathrm{mg} / 70 \mathrm{~kg}$ APREP, a GLM analysis was performed at the single-subject level. The purpose of the analysis was to determine whether a BOLD response could be captured by phMRI in regions with high densities 
of $\mu$-opioid or $\mathrm{NK}_{1}$ receptors due to $0.2 \mathrm{mg} / 70 \mathrm{~kg}$ BUP or $96 \mathrm{mg} / 70 \mathrm{~kg}$ APREP, respectively. The implemented design matrix comprised several explanatory variables (EVs). The main EV, modeling the effects of intravenous administration of BUP or APREP, consisted of a ramp function that followed the infusion paradigm for each drug. Owing to the long onset of the ramp functions relative to the hemodynamic response function (HRF), neither ramp function was convolved with an HRF. A linear drift term, along with subject-specific white matter, CSF, and head motion time courses, was also included in the design matrix as confound EVs. Furthermore, the time during the phMRI scan at which the BOLD signal was elevated from baseline in response to BUP or APREP could vary depending on structure or subject. To enable a robust and unbiased modeling of the infusion response while capturing this temporal variability, three additional regressors were created and included as covariates in the GLM analysis (Pendse et al, 2010b). The same BUP and APREP GLM models were utilized to analyze the respective saline data sets.

Single-subject functional connectivity analysis of phMRI data. To determine how BUP and APREP administration affects functional connectivity patterns within the brain circuitry, seed region functional connectivity analysis was performed (Fox and Raichle, 2007). Each BUP, APREP, and saline infusion data set was band-pass-filtered between 0.01 and $0.1 \mathrm{~Hz}$, thus removing the slow baseline infusion response and high-frequency noise (Cordes et al, 2001). Seed regions corresponding to the putamen and thalamus (each bilateral) were defined by using the AAL atlas (Tzourio-Mazoyer et al, 2002). To capture how the functional connectivity among various regions of interest (ROIs) was altered following BUP, APREP, or saline infusion, each data set was temporally segmented into four 5-min time blocks: (i) Min 0-5 (baseline, no drug exposure); (ii) $\min$ 5-10 (initial drug exposure); (iii) $\min 10-15$ (intermediate time during phMRI scan); and (iv) $\min 20-25$ (longest drug exposure during phMRI scan). From each 5 -min block, subject-specific time courses from the putamen and thalamus were extracted for seed region functional connectivity analysis. These regions were specifically chosen as structures with high densities of $\mu$-opioid (Greenwald et al, 2003; Zubieta et al, 2000) and $\mathrm{NK}_{1}$ (Bergstrom et al, 2004; Hietala et al, 2005) receptors. Furthermore, both structures are intimately involved in the various components of pain processing (sensory, motivational, and emotional) (Bingel et al, 2004; Maihofner et al, 2007; Singer et al, 2004). The white matter and CSF time courses were also extracted from each subject in each 5-min time interval and used as confound EVs in GLM analysis. Two sets of single-subject, whole-brain and GLM-based functional connectivity analysis were performed using either the putamen or the thalamus time courses.

Single-subject analysis of evoked pain fMRI data. The BOLD response elicited by noxious heat stimulation has been characterized previously as being biphasic, that is, having an early- and late-phase response with respect to the timing of the stimulus (Baliki et al, 2010; Becerra et al, 2001; Eippert et al, 2009; Upadhyay et al, 2010). Accordingly, the temporal BOLD response to the applied heat stimulus was modeled by using two EVs: a stimulus-locked regressor comprising the individual subject's applied stimulus, and a 12.5-s delayed version of this regressor (for details see Upadhyay et al, 2010).

Group-level analysis of phMRI and fMRI data. For all group-level image analysis, a mixed-effects (FLAME) paired $t$-test design was implemented (Woolrich et al, 2004). Multiple comparison corrections were performed by using a Gaussian mixture modeling (GMM) approach (Pendse $e t$ al, 2009). Details on the GMM analysis and thresholding are provided under Supplementary Materials and Methods. Briefly, 'null' and 'activation/deactivation' distributions were fit to the whole-brain, voxel-wise $z$-statistic distribution from each group comparison. The threshold selected for each corresponded to an activation/deactivation class posterior probability $(\mathrm{PP})>0.5$; that is, reflecting a higher probability that the supra-threshold voxels belonged to the activation/deactivation $v s$ the null class (Pendse et al, 2009). The actual threshold $z$ or $p$-value may thus differ slightly between data sets, but always reflect a constant $\mathrm{PP}>0.5$. Moreover, GMM explicitly models the data as a mixture of 'null' and 'activation' components, and makes inferences with respect to posterior probability of membership in the 'activation' class, thereby avoiding the multiple comparisons problem. Activation maps were also corrected by using Threshold-Free Cluster Enhancement (TFCE) (Smith and Nichols, 2009). In ROI summary statistic analyses as well in all non-imaging comparisons (eg, all bar and line graphs) we report two-tailed $p$-values.

\section{RESULTS}

Results for adverse effects, hedonic ratings, physiological measurements, and drug concentrations in plasma have been given in the Supplementary information (see also Supplementary Tables S1 and S2).

\section{Characterization of phMRI Infusion Responses for BUP and APREP}

Both BUP and APREP phMRI infusion responses were significantly distinct from the respective saline infusion responses. In Figure $1 \mathrm{a}$ and $\mathrm{b}$, the group-level $(n=12)$ phMRI infusion response for BUP $(0.2 \mathrm{mg} / 70 \mathrm{~kg}$ BUP minus saline) is shown, whereas in Figure $1 \mathrm{c}$ and d, group-level $(n=12)$ phMRI infusion responses are shown for APREP (96 mg/70 kg APREP minus saline). Administration of BUP $(p<0.02, \mathrm{PP}>0.5)$ and APREP $(p<0.05, \mathrm{PP}>0.5)$ yielded significant activation in regions with known distributions of $\mu$-opioid and $\mathrm{NK}_{1}$ receptors, respectively. For example, BUP-based activation was bilaterally observed in the striatum (caudate and putamen), thalamus, and anterior cingulate (Figure 1a). APREP-based activation likewise was bilaterally observed in the striatum and thalamus (Figure 1c). APREP phMRI infusion responses were detected in regions such as the cerebellum and red nucleus, where high densities of $\mathrm{NK}_{1}$ receptors do not exist. This result is likely a consequence of downstream effects of APREP. In Figure $1 \mathrm{~b}$ and $\mathrm{d}$, an ROI-level quantification of the responses measured within the putamen and thalamus 


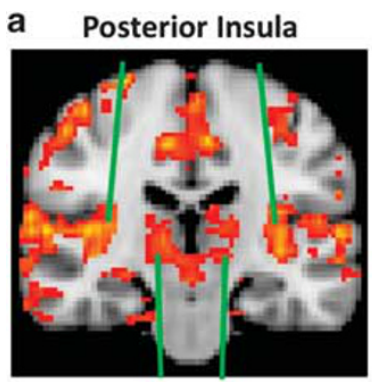

Thalamus

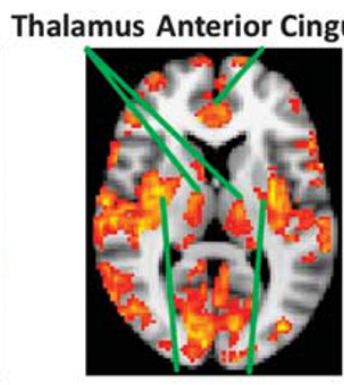

Putamen

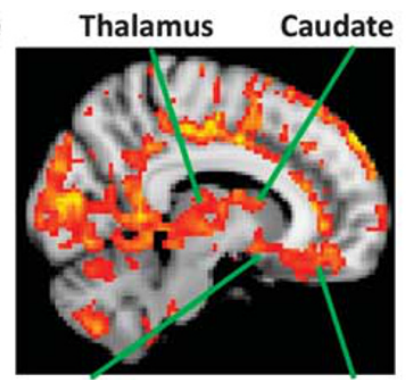

Nucleus
Frontal MidOrbital
Anterior Insula

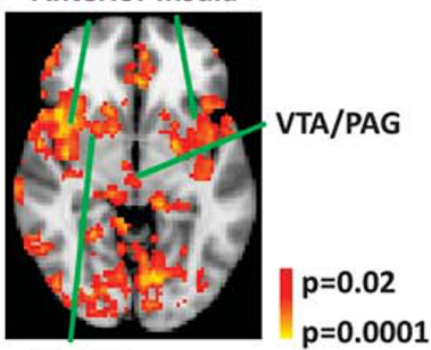

Globus

Pallidus

b
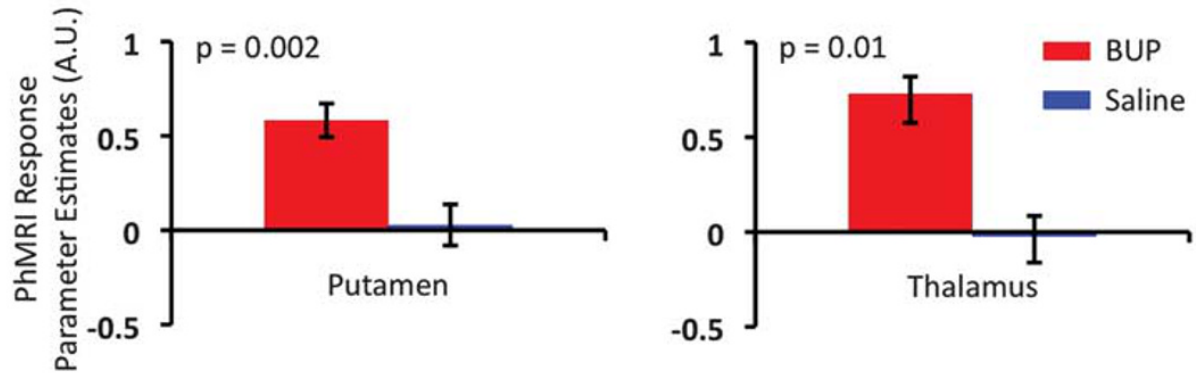

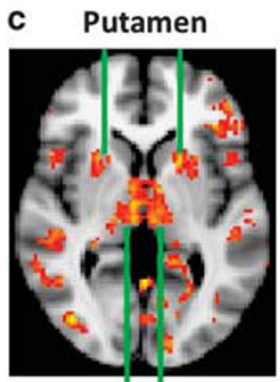

Thalamus

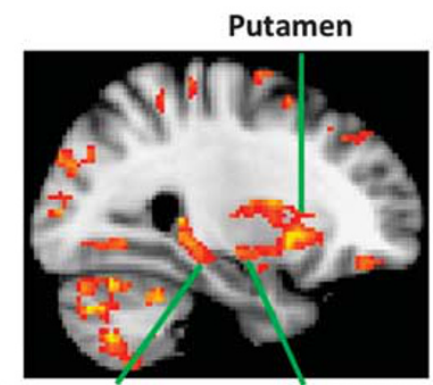

Hippocampus
Amygdala

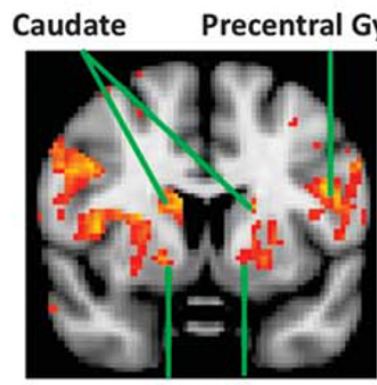

Globus

Pallidus

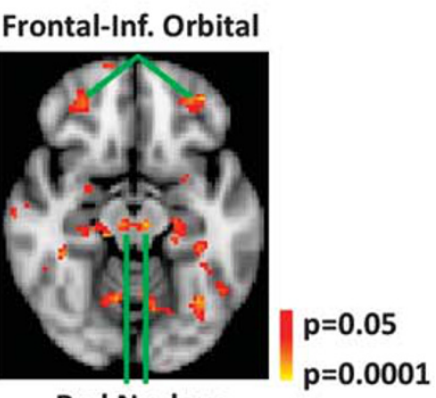

Red Nucleus

d
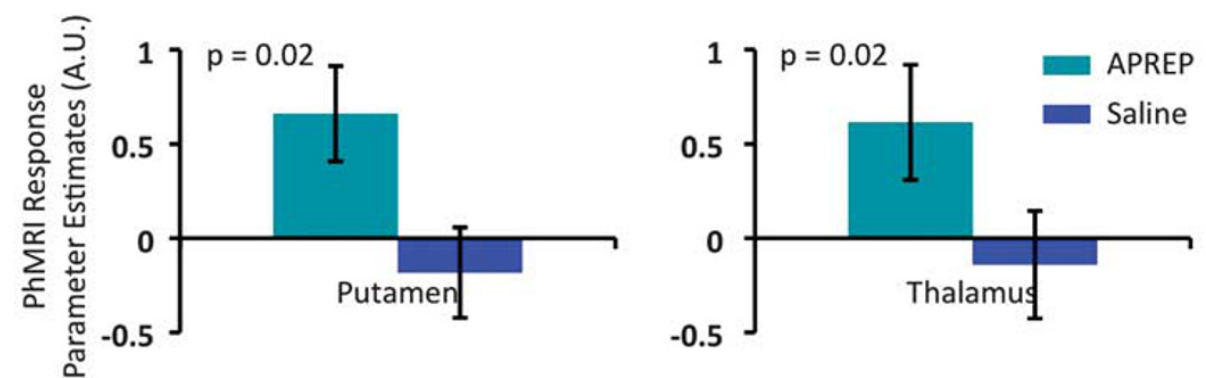

Figure I phMRI infusion response to $0.2 \mathrm{mg} / 70 \mathrm{~kg}$ BUP and $96 \mathrm{mg} / 70 \mathrm{~kg}$ APREP. (a) Group-level activation maps depict cortical and subcortical structures observed to have significantly $(p<0.02$, PP $>0.5)$ increased BOLD responses solely due to infusion of $0.2 \mathrm{mg} / 70 \mathrm{~kg}$ BUP. The BOLD activation maps shown in panel a are similar to PET-based $\mu$-opioid receptor distribution maps (Greenwald et al, 2003; Zubieta et al, 2000). (b) The parameter estimate values (mean \pm SE) calculated for the putamen and thalamus were significantly greater for the BUP group in comparison with the respective saline group. AU, arbitrary units. (c) Group-level activation maps depict cortical and subcortical structures observed to have significantly $(p<0.05, P P>0.5)$ increased BOLD responses solely due to infusion of $96 \mathrm{mg} / 70 \mathrm{~kg}$ APREP. The BOLD activation maps shown in panel a are similar to PET-based NK-I receptor distribution maps (Bergstrom et al, 2004; Hietala et al, 2005). (d) The parameter estimate values (mean \pm SE) calculated for the putamen and thalamus were significantly greater for the APREP group in comparison with the respective saline group. AU, arbitrary units.

is given for BUP and APREP, respectively. The putamen and thalamus parameter estimate values (mean \pm SEM) for BUP and APREP, as well as the respective saline data sets, were extracted from the anatomically defined putamen or thalamus. Additional quantification of BUP and APREP phMRI infusion responses is provided in Supplementary Tables S3 and S4, respectively.

\section{Characterization of BUP and APREP Effects on Functional Connectivity}

Sensorimotor circuitry functional connectivity changes. In comparison to the saline condition, several neuronal structures within the sensorimotor circuitry showed significantly decreased functional connectivity with the 
a

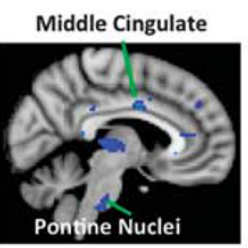

b
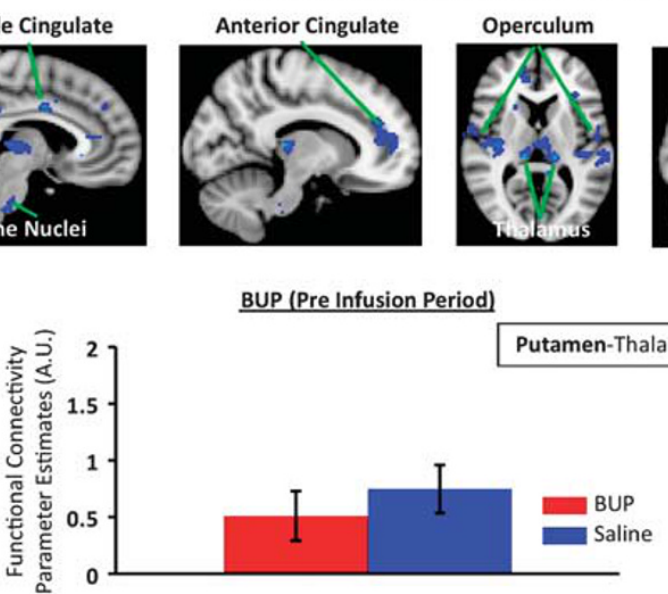

Functional Connectivity (Seed Region: Putamen): BUP (Post Infusion Period)
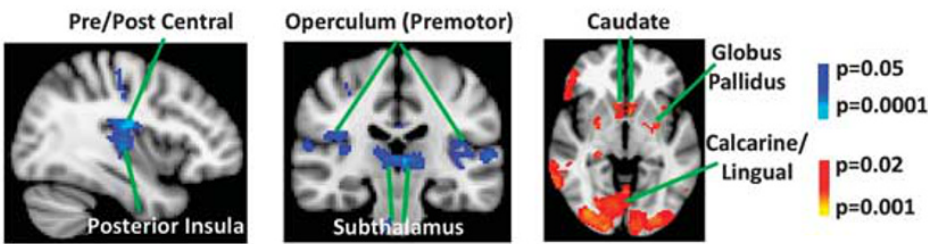

c

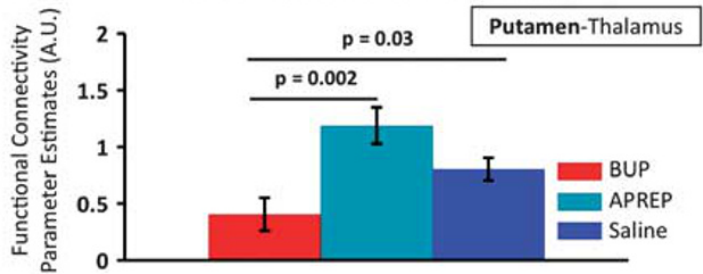

d Functional Connectivity (Seed Region: Thalamus): BUP (Post Infusion Period)

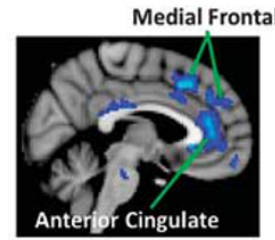

Caudate

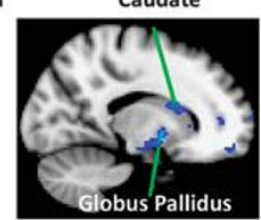

Amygdala

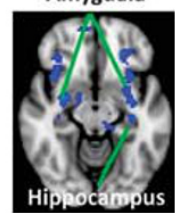

Inf. Orbital Frontal
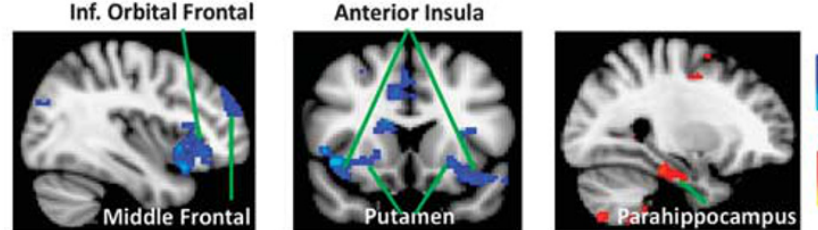

$p=0.05$

$p=0.0001$

e

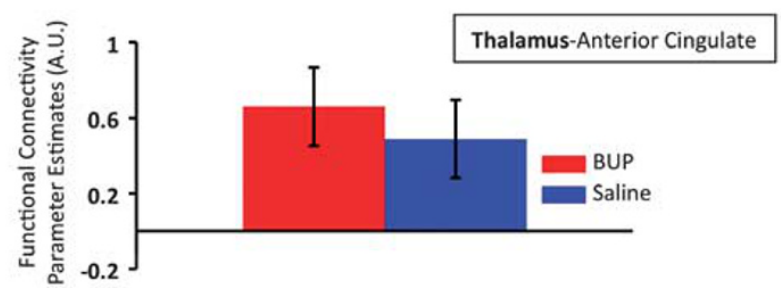

f

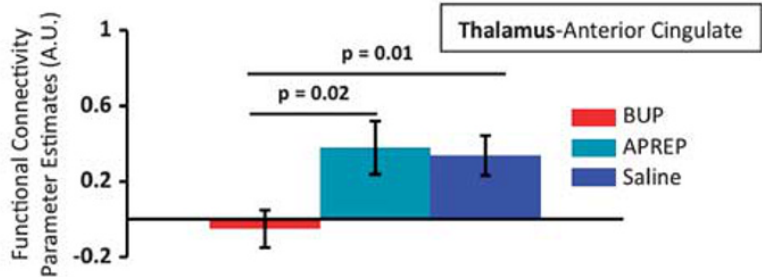

Figure 2 Functional connectivity in the sensorimotor circuitry and limbic circuitry under $0.2 \mathrm{mg} / 70 \mathrm{~kg}$ BUP treatment. (a) Cortical and subcortical structures were observed to have a significantly $(p<0.05$, PP $>0.5)$ decreased (blue-light blue) functional connectivity with the putamen or within the sensorimotor circuitry under $0.2 \mathrm{mg} / 70 \mathrm{~kg}$ BUP treatment. A significant increase (red-yellow) in functional connectivity with the putamen was also observed. (b) Group-level baseline parameter estimates representing functional connectivity during the pre-infusion period of the phMRI scan for the BUP and the respective saline data sets showed no significant difference in baseline connectivity between the putamen and thalamus. (c) Comparisons of functional connectivity differences between the putamen and thalamus across the $0.2 \mathrm{mg} / 70 \mathrm{~kg} B U P(n=12), 96 \mathrm{mg} / 70 \mathrm{~kg}$ APREP $(n=12)$, and saline $(n=24)$ cohorts are given. Characterization of functional connectivity between the putamen and thalamus is given as a quantitative example of changes occurring in the sensorimotor circuitry under BUP treatment. Parameter estimates were extracted from atlas-based (AAL atlas) definition of the thalamus. (d) Cortical and subcortical limbic structures were observed to have a significantly decreased $(p<0.05$, PP $>0.5$; blue-light blue) or increased ( $p<0.02$, PP $>0.5$; red-yellow) functional connectivity with the thalamus. (e) Baseline parameter estimates representing functional connectivity during the pre-infusion period of the phMRI scan for the BUP and the respective saline data sets showed no significant difference in baseline connectivity between the thalamus and anterior cingulate. (f) Comparisons of functional connectivity differences between the thalamus and anterior cingulate across the $0.2 \mathrm{mg} / 70 \mathrm{~kg} B U P(n=12), 96 \mathrm{mg} / 70 \mathrm{~kg}$ APREP $(n=12)$, and saline $(n=24)$ cohorts are given. Characterization of functional connectivity between the thalamus and anterior cingulate is given as a quantitative example of changes occurring in the limbic circuitry under BUP treatment. Parameter estimates were extracted from atlas-based (AAL atlas) definition of the thalamus. AU, Arbitrary units.

putamen under the $0.2 \mathrm{mg} / 70 \mathrm{~kg}$ BUP treatment (Figure $2 \mathrm{a}-\mathrm{c}$ ). In comparing functional connectivity results across the four 5-min time blocks the fourth time block (min 20-25) consisted of the most robust and significant changes in functional connectivity. Thus, the functional connectivity results from min 20-25 are reported in Figure 2. Structures that showed decreases in functional connectivity with the putamen are shown in blue-light blue (Figure 2a). Interestingly, the caudate and the globus pallidus both showed significant $(p<0.02, \mathrm{PP}>0.5)$ increases (depicted in redyellow) in functional connectivity with the putamen under
BUP treatment (Figure 2a). In Figure 2b, baseline parameter estimates were extracted from the pre-infusion period (first $5 \mathrm{~min}$ ) of the phMRI scan for both the BUP and the respective saline data sets. It is noted that that no significant difference in baseline connectivity of the putamen-thalamus functional interaction was present. Significant changes from putamen-based functional connectivity analysis were not observed in the APREP cohort. To further quantify the difference in functional connectivity of the putamenthalamus interaction across the three conditions (BUP, APREP, and saline), the group (mean \pm SEM) parameter 
estimates (extracted from the AAL atlas-based definition of the thalamus) are given in Figure $2 c$. Here it is shown that the BUP treatment condition is significantly decreased from the APREP and saline conditions. The saline condition parameter estimates reported in Figure $2 \mathrm{c}$ represent the average parameter estimates from the BUP and APREP subject cohorts $(n=24)$. Similarly, ROI analysis for other sensorimotor functional connections (eg, putamen-posterior insula connection) were also observed to have a significantly decreased connectivity only between the BUP and saline $(p=0.01)$ and not between the APREP and saline conditions.

Limbic circuitry functional connectivity changes. Significant decreases (blue-light blue) in limbic system functional connectivity were observed in the BUP as compared with saline condition where the thalamus was used as a seed region (Figure 2d). The parahippocampus was the only limbic structure that demonstrated a significant increase (depicted in red-yellow) in functional connectivity. Significant functional connectivity changes were not observed within the limbic circuitry under the APREP condition. No significant difference in pre-infusion functional connectivity between the thalamus and the anterior cingulate across the two conditions was detected in the BUP cohort (Figure 2e). To further quantify the difference in functional connectivity of the thalamus-anterior cingulate interaction across the three conditions (BUP, APREP, and saline), the group-level parameter estimate values (mean \pm SEM) are given in Figure 2f. Here, parameter estimates were extracted from the AAL atlas-based definition of the anterior cingulate. ROI analysis similar to Figure $2 \mathrm{f}$ for other limbic functional connections (eg, thalamus-anterior insula connection) also showed significant decreases in connectivity only between the BUP and saline conditions $(p=0.05)$. A Pearson correlation analysis of the change in thalamusanterior cingulate functional connectivity and change in pain ratings collected during the subsequent noxious heat stimulation between BUP and saline treatment conditions revealed a significant relationship $(R=0.65, p=0.02)$, with greater changes in functional connectivity associated with increased changes in pain ratings.

Dependence of functional connectivity changes on BUP concentration in plasma. By using the subject-specific peak BUP concentration values as regressors of interest in grouplevel analysis, we observed a significant relationship between the two measures. Brain regions showing postBUP vs post-saline functional connectivity changes were also negatively correlated with peak BUP plasma concentration. For the putamen seed, these regions included the postcentral gyrus and the operculum (bilaterally) (Figure 3a), and for the thalamus seed, the anterior insula and the anterior cingulate (Figure $3 \mathrm{~b}$ ).

\section{Consistency of BOLD Response and Pain Ratings to Noxious Heat Stimuli}

The data from the BUP and APREP cohorts each included $n=12$ data sets acquired in the presence of placebo (physiological saline only). To determine the consistency of the early- and late-phase BOLD responses to noxious heat across the BUP and APREP subject cohorts as well as the consistency of VAS pain ratings, a group-average, mixedeffects, unpaired comparison was performed between the saline data sets in each cohort. No significant differences were measured for early- and late-phase BOLD responses or for pain ratings $(p=0.58)$ between the two saline groups. Significant differences for early-phase BOLD responses were not detected in pain regions such as the thalamus $(p=0.23)$ or the nucleus accumbens $(p=0.40)$ between the two saline data sets. Similarity between the two saline data sets for latephase responses was also detected in pain regions such as the anterior insula $(p=0.50)$ or the caudate $(p=0.93)$. To further demonstrate the consistency of BOLD responses to noxious heat across the two experimental cohorts, the mixed-effects, within-group average as well as the mixedeffects, unpaired comparison (saline (BUP cohort) vs saline (APREP cohort)) results have been given in the Supplementary information. The consistency of BOLD fMRI responses and pain ratings between the two saline cohorts strongly suggested the robustness of the two measures across cohorts.

\section{Effects of BUP and APREP on the BOLD Response to Noxious Heat Stimuli}

$0.2 \mathrm{mg} / 70 \mathrm{~kg}$ BUP. In comparison to the saline condition, BUP treatment potentiated the early-phase response in the limbic and mesolimbic circuitry (Figure 4a). Thus, brain regions mediating emotional and motivational components of pain were potentiated by BUP. It is noted that for defaultmode network (DMN) structures such as the anterior/ perigenual and posterior cingulate/precuneus, we observed a change in valence for the BOLD response, with the negative response in the saline condition becoming positive following BUP administration. In contrast to structures within the limbic circuitry and the DMN, structures of the sensory-discriminative or sensorimotor circuits (including mediodorsal/centromedian thalamus, primary/secondary somatosensory cortices, and posterior insula) were observed to have an attenuated late-phase BOLD response to noxious heat subsequent to BUP administration (Figure 4a). During pain fMRI scans where BUP was present, plasma BUP concentration was between $0.53 \pm 0.03$ (blood draw at $45 \mathrm{~min}$ ) and $0.55 \pm 0.03 \mathrm{ng} / \mathrm{ml}$ (blood draw at $85 \mathrm{~min}$ ). Additional quantification of early- and late-phase BOLD responses to noxious heat for the BUP cohort is provided in Supplementary Table S5.

$96 \mathrm{mg} / 70 \mathrm{~kg} A P R E P$. In comparison to the effects of BUP, the effects of APREP on the BOLD response to noxious heat pain were much less widespread. APREP treatment potentiated the early- and late-phase BOLD response in the limbic circuitry (eg, anterior cingulate, amygdala, and inferior orbital frontal cortex) but also in sensorimotor regions such as the Supplemental motor cortex (Figure $4 \mathrm{~b}$ ). During pain fMRI scans where APREP was present, the APREP concentration was between $1806.33 \pm 169$ (blood draw at $40 \mathrm{~min}$ ) and $1225.17 \pm 85 \mathrm{ng} / \mathrm{ml}$ (blood draw at $85 \mathrm{~min}$ ). Additional quantification of early and late phase BOLD responses to noxious heat for the APREP cohort has been given in Supplementary Table S6. 


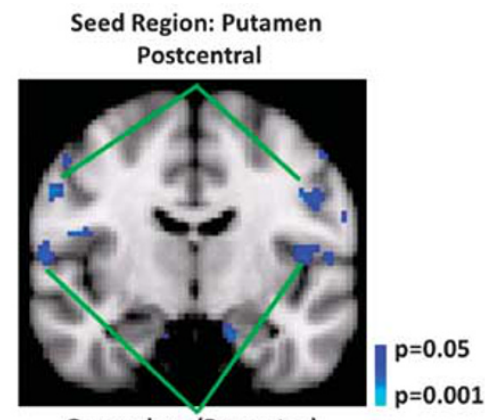

Operculum (Premotor)

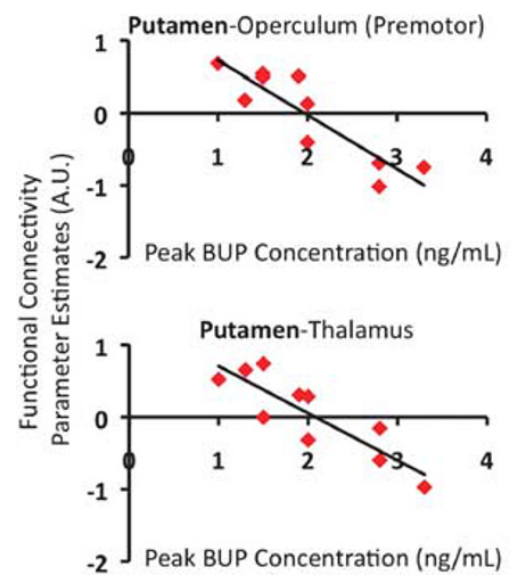

b
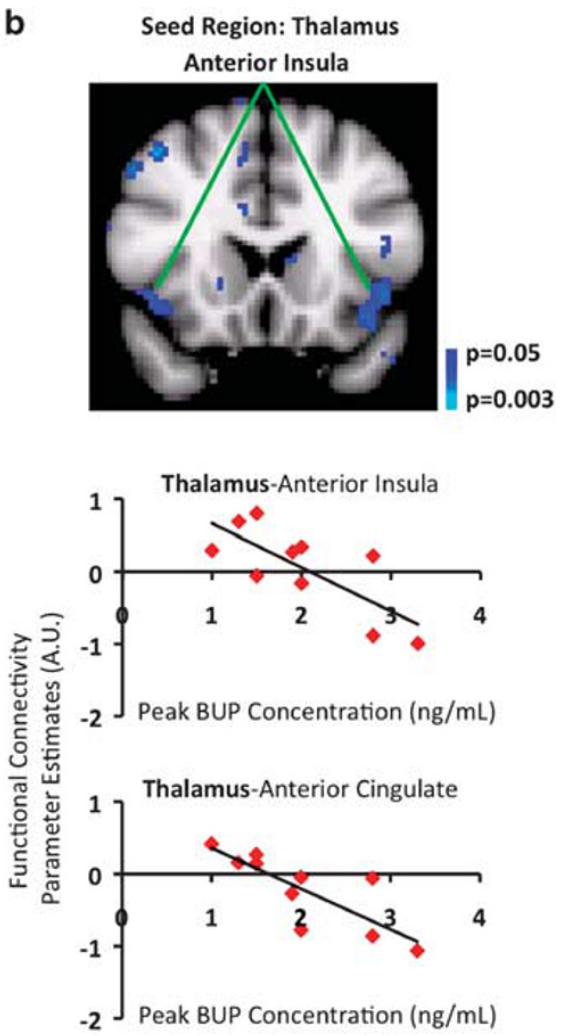

Figure 3 Dependence of functional connectivity changes on peak BUP concentration in plasma. Functional connectivity alterations occurring in the sensorimotor (Figure $2 \mathrm{a}-\mathrm{c}$ ) and limbic (Figure $2 \mathrm{~d}-\mathrm{f}$ ) circuitry were observed to have a significant $(p<0.05, \mathrm{PP}>0.5)$ dependence on peripheral peak BUP concentration. In prior analysis and results, functional connectivity decreases were observed between the putamen and thalamus, and also between the putamen and postcentral gyrus as a result of BUP treatment. (a) Inclusion of peak BUP concentrations in group-level analysis showed that the higher the peak BUP concentration, the lower the functional connectivity (defined by parameter estimates) between the putamen and thalamus or between the putamen and postcentral gyrus. (b) Likewise, previous analysis revealed decreased functional connectivity between the thalamus and anterior insula or between the thalamus and anterior cingulate. Here it was also observed that the higher the peak BUP concentration, the lower the functional connectivity. AU, Arbitrary units.

\section{VAS Pain Ratings to Noxious Heat Stimuli}

In comparison to the saline condition, BUP treatment produced a significant $(p=0.03)$ group-level decrease in VAS pain ratings to noxious heat, whereas APREP did not $(p=0.70)$. For the BUP cohort $(n=12)$ the mean \pm SEM pain rating was $4.33 \pm 0.63$ (BUP) and $6.81 \pm 0.86$ (saline). For the APREP cohort $(n=12)$ the mean \pm SEM pain rating was $6.00 \pm 0.93$ (APREP) and $6.19 \pm 0.72$ (saline).

\section{Effect Size Comparison}

The effect size of BOLD fMRI and VAS pain rating data was compared within each cohort (drug vs saline) (Figure 5). Effect sizes for BOLD fMRI data were calculated for four ROIs that are known to have a role in pain processing. In each of the comparisons (BUP vs saline and APREP $v s$ saline), BOLD fMRI effect size values were observed to be consistently greater in comparison with VAS pain ratings effect sizes, whereas the greatest effect was consistently observed for the BUP cohort (BUP vs saline; 0.75-1.24). The APREP cohort (APREP $v s$ saline) demonstrated small-tomoderate effects for BOLD fMRI and VAS data (0.07-0.45). The effect sizes for BOLD fMRI data were calculated by using parameter estimates extracted from atlas-defined ROIs rather than from regions of a structure observed to have significant potentiation or attenuation. Thus, statistically insignificant voxels were incorporated in the BOLD fMRI effect size calculation. Even so, the BOLD fMRI effect size was greater than that for VAS pain ratings.

\section{Correlation of BOLD fMRI with VAS Pain Ratings}

In the BUP cohort, significant attenuation of the late-phase BOLD response to noxious heat was observed in the somatosensory circuitry (Figure 6a and b). Specifically, the contralateral primary somatosensory cortex, a structure known to encode pain intensity (Coghill et al, 1999), showed a significant attenuation of the late-phase BOLD response under BUP treatment. By contrast, the APREP treatment condition did not significantly alter the BOLD response in comparison with the saline condition. For the BUP cohort, a significant correlation (Pearson correlation analysis) between pain ratings and the late-phase BOLD response was measured (Figure 6c). For BUP, similar correlation patterns in other sensory or sensorimotor structures (eg, bilateral posterior insula: $R=0.62$, $p=0.001$; bilateral thalamus: $R=0.52, p=0.009$ ) were also observed. Regions such as the nucleus accumbens, whose early-phase BOLD response was significantly potentiated subsequent to BUP treatment (Figure 4), were found to have 

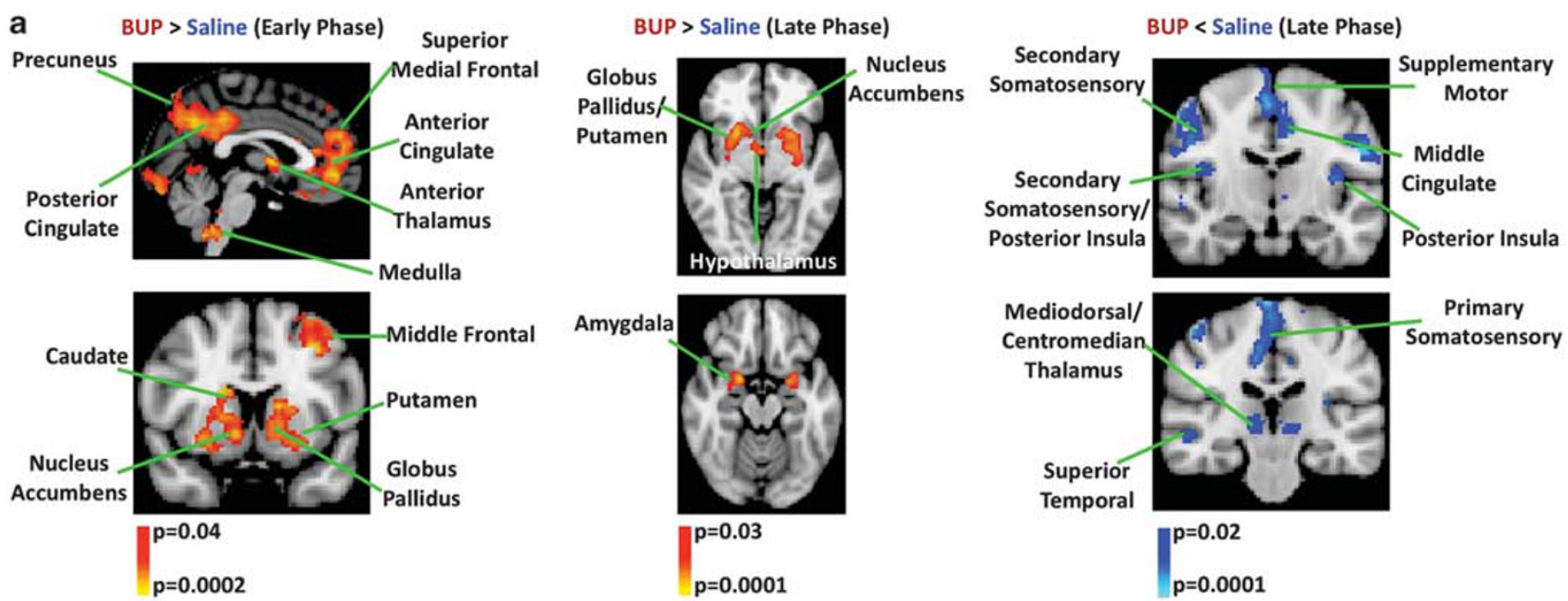

b APREP > Saline (Early Phase)
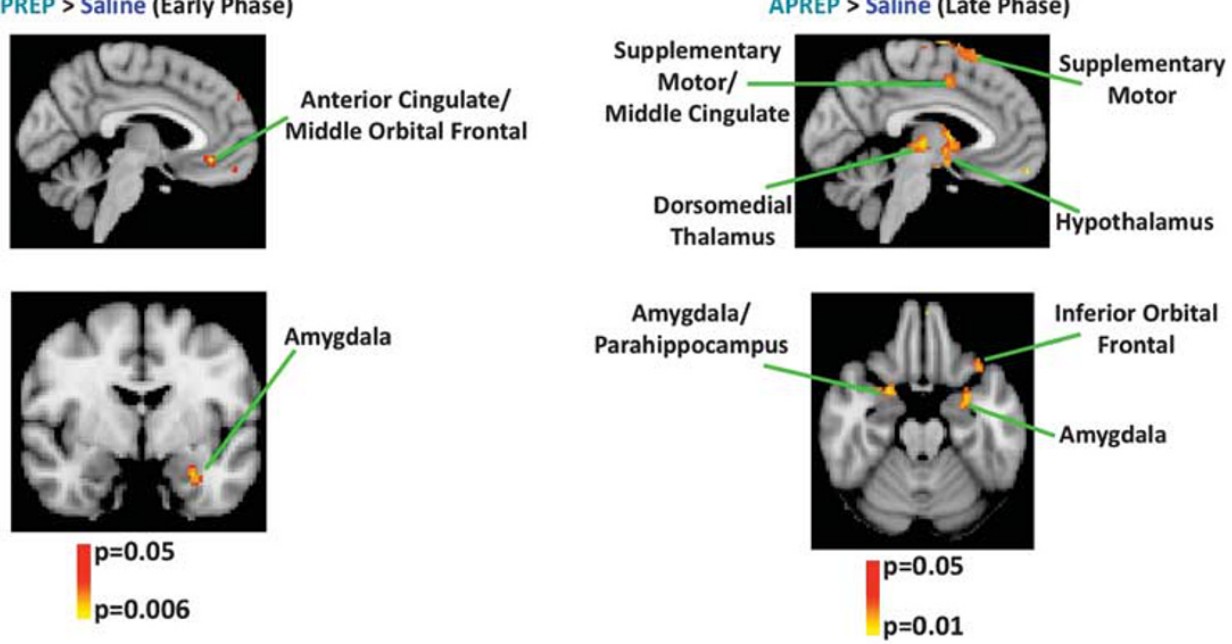

Figure 4 Modulation of BOLD response to noxious heat stimuli in the limbic and sensorimotor circuitry by BUP and APREP. Implementing a two-EV GLM analysis enabled a characterization of the early- and late-phase BOLD responses elicited by noxious heat stimulation. Whereas the early-phase response was locked to the heat stimulus, the late-phase response was delayed by I2.5 s from stimulus onset (Upadhyay et al, 20 I0). (a) Group analysis revealed a significant $(P<0.04, \mathrm{PP}>0.5)$ potentiation (BUP $>$ saline) of the early-phase BOLD response in limbic structures owing to BUP administration as well as within the DMN. Significant attenuation (BUP < saline) of the early-phase BOLD response was not observed. BUP yielded a significant ( $p<0.03$, $\mathrm{PP}>0.5)$ group-level potentiation of the late-phase BOLD response to noxious heat in regions such as the dorsal/ventral striatum and amygdala, whereas a significant $(p<0.02, \mathrm{PP}>0.5)$ attenuation of the late-phase BOLD response was identified in sensory and sensorimotor structures such as the primary/ secondary somatosensory and supplemental motor cortices. (b) The two-EV GLM analysis revealed a significant potentiation during the early ( $p<0.05$, PP $>0.5)$ and late $(p<0.05, P P>0.5)$ phases of the BOLD response owing to APREP administration. Significant attenuation of early- or late-phase BOLD responses was not observed. The potentiation in the early (a) and late (b) phases was predominately observed in limbic (ie, amygdala, inferior orbital frontal, and subgenual anterior cingulate) and sensorimotor (supplemental motor and supramarginal cortices) structures. In comparison with BUP, the modulatory effects of APREP on the BOLD response to noxious heat stimuli were much less robust.

a significant inverse correlation with VAS pain ratings (contralateral nucleus accumbens: $R=-0.44, p=0.03$ ).

\section{Correlation of BOLD fMRI with Drug Concentration in Plasma}

The difference in the BOLD response to the noxious heat stimulus between the BUP and saline conditions showed a significant correlation with peak BUP concentration in plasma in the anterior cingulate and prefrontal cortex (Figure 7a). These colocalized with brain regions that evidenced potentiated responses per se to the stimulus (Figures 4 and 7a).

\section{Summary of BUP Induced Changes in the CNS Pain Circuitry}

From phMRI and fMRI data a number of pharmacodynamic changes were observed in the CNS pain circuitry owing to BUP administration. The following changes were observed in the sensorimotor (Figure 8a) and limbic (Figure 8b) circuitry: (i) Significant phMRI infusion response to BUP; (ii) decreased functional connectivity with the putamen or thalamus; and (iii) attenuation or potentiation of evoked BOLD responses elicited by noxious heat stimulation. Using the Oxford Thalamic Connectivity Probability Atlas (www.fmrib.ox.ac.uk/fsl), the thalamic regions observed to 
have phMRI and fMRI-based pharmacodyamic changes corresponded to those projecting to sensory, motor, and prefrontal cortices (Figure 8a).

\section{DISCUSSION}

The current study focused on a phMRI- and fMRI-based comparison of two pharmacological compounds developed as analgesics, where one was successful and the other failed for this indication. This study reports on a number of novel findings, including phMRI of the opioid BUP, phMRI of the $\mathrm{NK}_{1}$ antagonist APREP, and objectively characterizing effective pharmacology underlying an analgesic effect by using functional connectivity analysis and an evoked pain fMRI. Currently, in preclinical and early-phase clinical analgesic drug development, there is a pressing need for

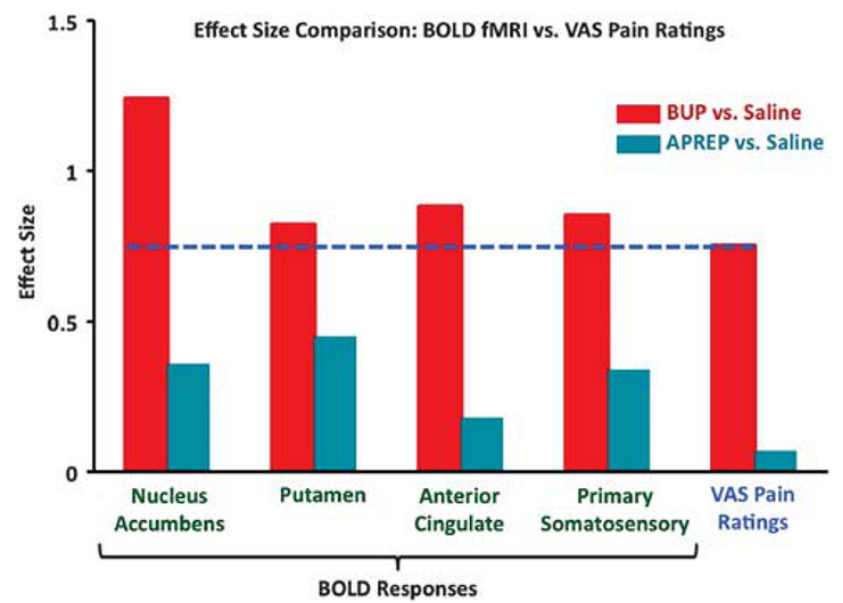

Figure 5 Higher effect size values for BOLD fMRI than VAS pain rating data. Effect size values greater than 0.5 were considered to be large effects, whereas values between 0.1 and 0.5 were small-to-moderate effects (Cohen, 1988). BOLD fMRI effect size values are higher than VAS pain rating effect sizes in each of the comparisons. Whereas the greatest effect for both BOLD FMRI and VAS data was consistently observed for the BUP cohort (BUP vs saline), the APREP cohort (APREP vs saline) demonstrated smallest effects. The blue line demonstrates that the VAS pain rating effect size specific to the BUP cohort was consistently lower than the BOLD fMRI effect sizes for the four ROls. additional tests and tools that help determine which CNS mechanisms of action(s) or targets are best to engage in order to yield effective analgesia. With an improved understanding of these CNS mechanisms of action(s) or targets, the likelihood of identifying clinically effective analgesic drugs may be enhanced. The results of this work suggest that the combination of phMRI and fMRI measures in small cohorts of healthy volunteers may contribute to this end. For therapeutic domains such as pain, there is a pressing need for methodology and biomarkers that are translatable between species and studies. phMRI and fMRI are two methods that may be implemented to obtain translatable biomarkers for pain.

\section{Measurement of phMRI Infusion Responses and Pharmacological Modulation of Functional Connectivity}

Previous phMRI studies have assessed the feasibility of implementing BOLD fMRI techniques as a means to define regions of increased activity in the human brain solely due to intravenous administration of a drug (Becerra et al, 2006; Borras et al, 2004; Leppa et al, 2006). Here, we have extended these approaches and characterized the phMRI infusion responses for a $\mu$-opioid receptor partial agonist and an $\mathrm{NK}_{1}$ receptor antagonist, and found overlap between the two phMRI results as well as with previously reported receptor-occupancy data for each drug (Figure 1). For example, Greenwald et al (2003) and Zubieta et al (2000) previously reported high occurrence of BUP- $\mu$-opioid receptor binding in regions such as the striatum (caudate/ putamen), thalamus, anterior cingulate, and prefrontal cortex. Likewise, phMRI infusion responses for BUP were also detected in these same structures, and were similar to previous phMRI investigations involving other opioids such as morphine, naloxone, or remifentanil (Becerra et al, 2006; Borras et al, 2004; Leppa et al, 2006).

The two drugs differed more robustly in how functional connectivity was pharmacologically modulated. Only BUP significantly modulated functional connectivity in the CNS circuitry relevant to pain processing (ie, sensorimotor and limbic) (Figure 2), with changes in these regions also dependent on plasma BUP concentration (Figure 3). The decreases in functional connectivity are postulated to result a

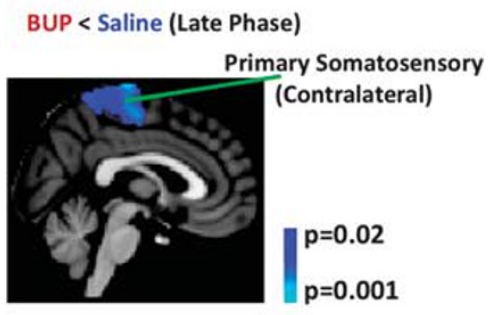

b

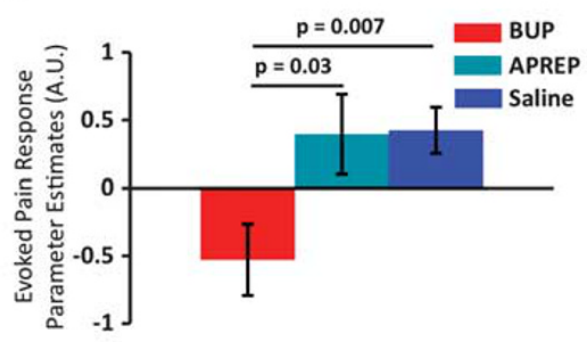

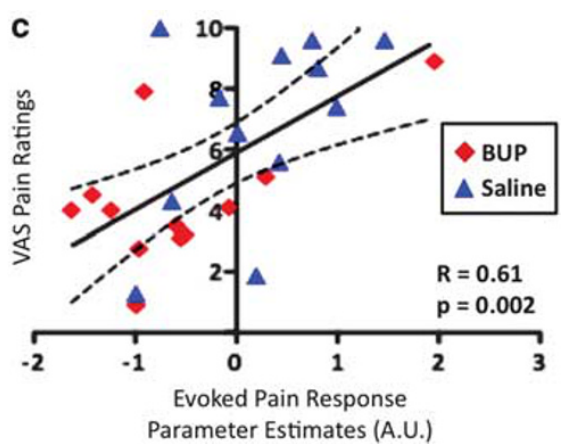

Figure 6 BOLD $\mathrm{AMRI}$ vs VAS pain ratings to differentiate drug effect. (a) BUP attenuated the late-phase BOLD response in the contralateral primary somatosensory cortex elicited by noxious heat stimulation. (b) Parameter estimates were extracted from the contralateral primary somatosensory cortex in order to perform a group comparison. The primary somatosensory cortex was anatomically defined by using the AAL atlas and also based on previous work by Nakamura et al (1998). Parameter estimates corresponding to the saline condition were pooled together from the BUP and APREP cohorts. No statistical difference in parameter estimate values was detected between the two saline groups. (c) A significant correlation between VAS pain ratings and the late-phase BOLD responses (parameter estimates) from the contralateral primary somatosensory cortex was measured for the BUP cohort. 

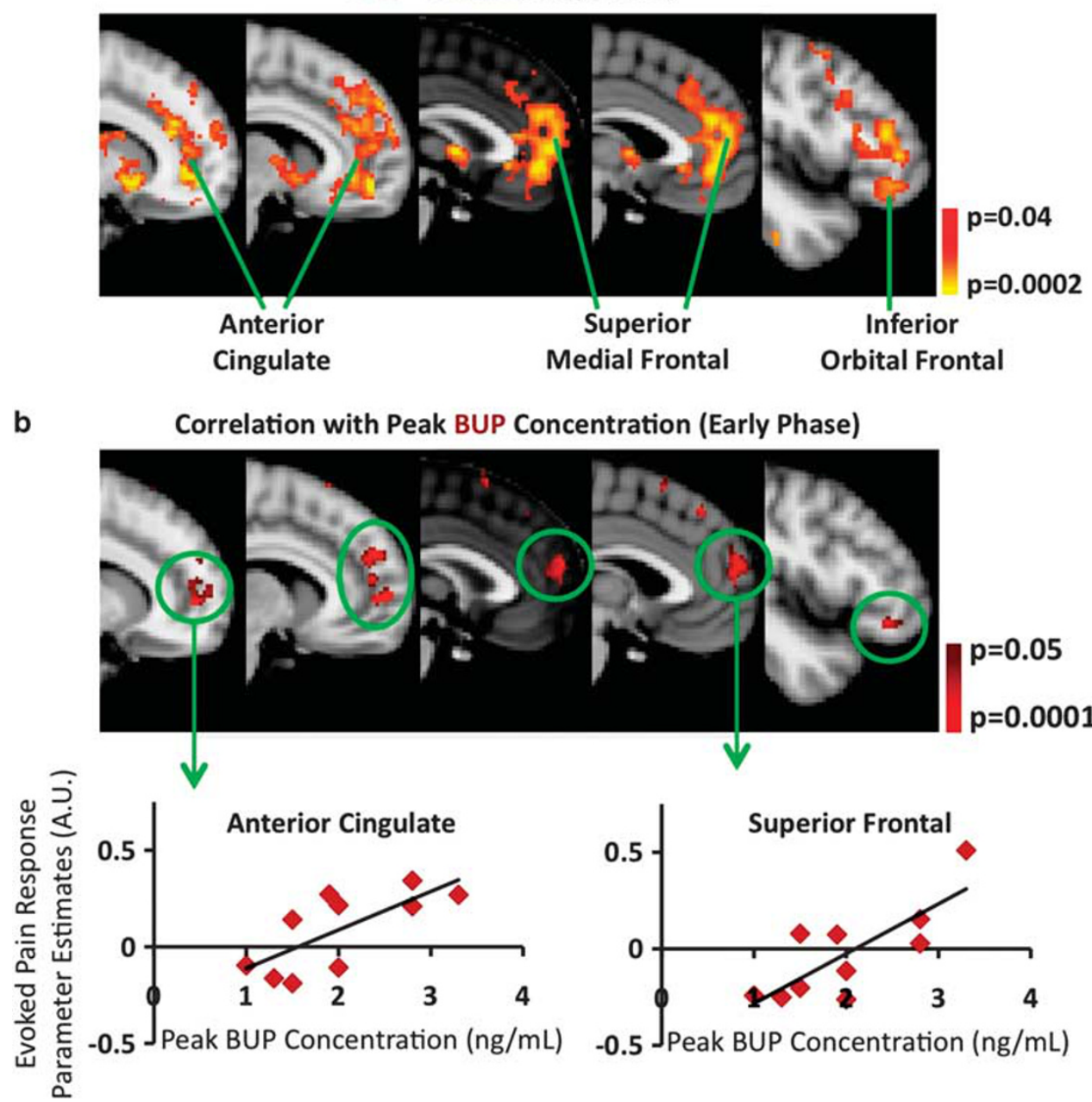

Figure 7 Dependence of BOLD FMRI responses to noxious heat on peak BUP plasma concentration. (a) BUP potentiated the early-phase BOLD response to noxious heat in the anterior cingulate, superior frontal, and inferior orbital frontal cortices. (b) Inclusion of peak BUP concentrations in grouplevel analysis showed that the higher the peak BUP concentration, the higher the BOLD response in the anterior cingulate and frontal cortex regions. Using functional ROls, parameter estimates were extracted from two regions to further quantify the relationship between the BOLD response and BUP plasma concentration.

from the disruption of GABA- and glutamate-mediated processes, which are heavily present in the sensorimotor and limbic system circuitry (Fingelkurts et al, 2004). As with the phMRI infusion responses, the modulation of functional connectivity due to BUP administration is likely to be a combination of pharmacological effects in regions with high densities of $\mu$-opioid receptors as well as downstream structures (see Supplementary information for study limitations). Moreover, whether and how a pharmacological compound modulates connectivity in an entire brain system may be important to determine. The latter finding on functional connectivity suggests that, although a novel pharmacological compound reaches specific neuronal targets through a drug-receptor binding, how the drug perturbs functional interactions in the relevant brain circuitry may be important to consider as an additional measure. Such approaches have been reported in animal models to differentiate drug effects (Schwarz et al, 2009).

The perturbations of functional connectivity in the sensorimotor and limbic circuitry subsequent to BUP administration is salient as these systems mediate distinct yet interrelated components of pain processing. Pain consists of sensory, motivational, and emotional components, each of which contributes to the overall experience of pain. Moreover, there is also an overlap in structures that are part of either the sensorimotor or the limbic circuitry, where a single anatomical structure (ie, insula may be functionally segregated (eg, anterior insula-emotional processing (Phan et al, 2002); posterior insula-motor processing (Fink et al, 1997)). Thus, a substantial pharmacological perturbation of one circuitry system of pain is likely to affect another, yielding a change in the perception of pain. Given the known role of the thalamus and putamen in the sensory and affective components of pain, we used these brain structures as seed regions in functional connectivity analysis. Although seed regions included only the putamen and thalamus, these structures are well connected to other pain-processing structures, as well as other cortical and subcortical structures expressing either $\mu$-opioid or $\mathrm{NK}_{1}$ receptors. Moreover, the pharmacological modulation in functional connectivity by BUP 


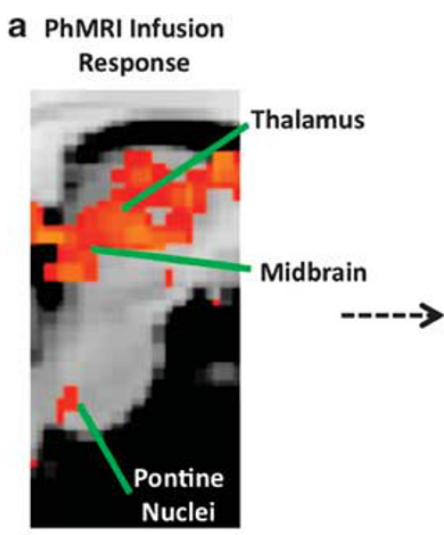

\section{Decreased Functional}

Connectivity with Putamen

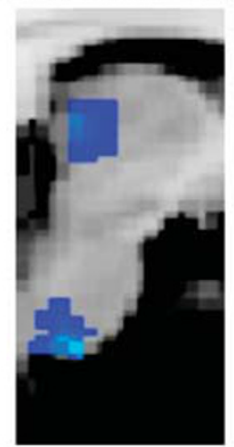

Attenuation of BOLD

Response to Noxious Heat

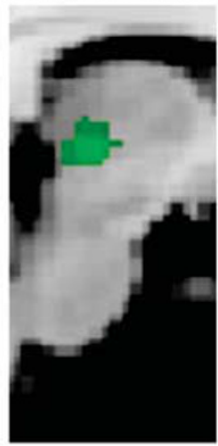

b PhMRI Infusion Response

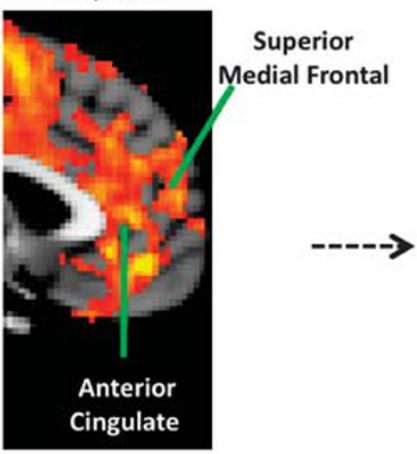

$0.2 \mathrm{mg} / 70 \mathrm{~kg}$ BUP vs. Saline ( $\mathrm{N}=12$ )
Decreased Functional Connectivity with Thalamus

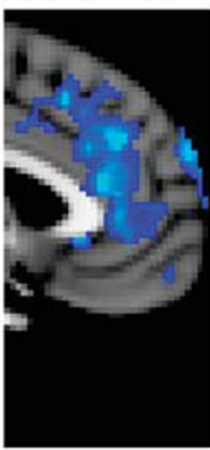

$0.2 \mathrm{mg} / 70 \mathrm{~kg}$ BUP vs. Saline ( $N=12)$
Potentiation of BOLD

Response to Noxious Heat
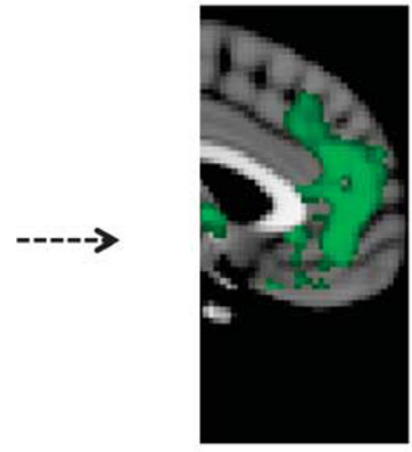

$0.2 \mathrm{mg} / 70 \mathrm{~kg}$ BUP vs. Saline ( $N=12)$

Figure 8 Relationship among phMRI, functional connectivity, and evoked pain fMRI measures. Sensorimotor (a) and limbic (b) circuitry structures were measured to be pharmacologically modulated by BUP from three distinct functional imaging perspectives: phMRI infusion response, functional connectivity, and evoked BOLD response to noxious heat. Functional connectivity and BOLD responses to evoked pain were related to non-imaging-based measured such as pharmacokinetic $\left(C_{\max }\right)$ quantities and subjective pain ratings.

(eg, thalamus-anterior cingulate functional interaction) was concomitant with a reduction in perceived pain subsequently collected during the evoked pain state.

\section{Modulation of Early- and Late-Phase CNS Responses to Evoked Pain by BUP and APREP}

Robust modulation of CNS responses to evoked pain by $B U P$. The implemented fMRI analysis enabled a characterization of the early- and late-phase BOLD responses elicited by noxious heat stimulation. Significant potentiation was observed within limbic or mesolimbic circuits (Figure 4a), which are known to mediate the emotional, motivational, and attention aspects of pain (Becerra et al, 2001). This potentiation in regions such as the cingulate cortex was correlated with BUP concentration in plasma (Figure 7). Specifically, our data show that under the BUP condition, the activity in the DMN, a network consisting of limbic structures (eg, cingulate), is potentiated, or the DMN remains in a more active state in comparison with the saline condition (Figure 4a). Previous studies have shown that in healthy subjects, the DMN significantly deactivates during sensory processing and focused mental activity (Baliki et al, 2008). The potentiation of the DMN by BUP may be related to a lack of focused mental activity to the noxious heat stimuli. In the context of chronic pain, the particular modulation of the DMN by BUP is noteworthy given the connectivity changes observed in or with this particular network in diseases such as chronic back pain (Baliki et al, 2008) or fibromyalgia (Napadow et al, 2010).

It is known that limbic structures such as the cingulate, amygdala, ventral/dorsal striatum, or hypothalamus contain high levels of $\mu$-opioid receptors and endogenous opioids in both rodents (Mansour et al, 1988) and humans (Greenwald et al, 2007). Our findings are in accord with preclinical data that clearly show that agonist activity at the $\mu$-opioid receptor also in limbic regions yields an inhibition of behavioral responses to noxious stimulation (Manning et al, 1994; Tershner and Helmstetter, 2000; Yaksh and Rudy, 1978). This investigation in conjunction with previous clinical and preclinical studies suggests that activation of these limbic structures containing $\mu$-opioid receptors facilitates analgesia. The agonist effect on the $\mu$-opioid system during a painful event has been linked to alterations in the processing of the aversive quality of pain (LaGraize et al, 2006; Zubieta et al, 2001).

Considering previous investigations involving morphine, also a $\mu$-opioid agonist, the potentiation caused by BUP observed in brain stem structures such as the medulla (Figure 4a) may in part be derived from the pre- and 
postsynaptic inhibition of GABA release, which in turn leads to excitatory activity of off cells in such regions (Heinricher et al, 1992; Vaughn et al, 1997). In rats subjected to morphine administration and noxious stimulation, enhanced off-cell activity occurs simultaneously with suppression of on-cell activity (Heinricher et al, 1994). Thus, the potentiation in structures that are part of the descending pain circuitry likely contributes to the analgesic effect observed under BUP administration.

Conversely, the evoked BOLD response in structures within sensory-discriminative and sensorimotor circuits was attenuated following BUP treatment (Figure 4a). Specifically, the somatosensory, posterior insular, supplemental motor, and thalamic regions were observed to have a significant attenuation of the late-phase BOLD response. Preclinical work has shown a suppression of thalamic noiciceptive activity following $\mu$-opioid receptor agonist action (Benoist et al, 1983; Yang et al, 1998). The relationship observed between the attenuation of the BOLD response within sensory-discriminative and sensorimotor structures, and attenuation in pain ratings provides an important link between CNS function and behavioral output (Figure 6).

BUP is a non-selective, centrally acting opioid, with partial agonist activity at the $\mu$-and $\delta$-opioid receptor site, and antagonist activity at $\kappa$-opioid receptors (Lutfy et al, 2003). However, in the context of BUP-induced analgesia, the partial agonist action on $\mu$-opioid receptors is believed to have a dominant role (Ide et al, 2004; Lutfy et al, 2003). Ide et al have shown that BUP-induced analgesia was diminished in heterozygous $\mu$-opioid receptor-knockout mice, whereas abolished in the homozygous strain. With regard to $\delta$ - and $\kappa$-opioid receptors, the effects of BUP were more associated with reward processes. Given that reward and aversion are integral components of pain (Baliki et al, 2010; Becerra et al, 2001), and $\kappa$-opioid receptor agonists produce aversive behaviors (Sante et al, 2000), the agonist activity at $\mu$ - and $\delta$-opioid receptors in conjunction with antagonist action at $\kappa$-opioid receptors by BUP collectively contribute to the BOLD fMRI response patterns and the analgesic effect observed in the current study.

Similar attenuation of analgesic effects in $\mu$-opioidknockout mice has been observed with other opioids such as morphine (Ide et al, 2004; Sora et al, 2001). Moreover, as in the current study where phMRI infusion responses were measured in the limbic and mesolimbic circuitry, phMRI studies of morphine yielded similar activation patterns (Becerra et al, 2006). It is noted that phMRI infusion analysis procedures did vary slightly between the previous and the current study, and may contribute to the differences observed between the two sets of results. Moreover, in a recent study by Khalili-Mahani et al (2011), increases in cerebral blood flow were observed in the limbic and sensory regions following administration of morphine. This previous finding is also in line with the current phMRI results of BUP.

Effects on early-and late-phase CNS responses to evoked pain by APREP. APREP yielded a subtle yet significant potentiation of early- and late-phase BOLD responses within limbic structures such as the amygdala, middle frontal cortex, and orbital frontal cortex (Figure 4b). APREP administration did not result in a significant attenuation of early- or late-phase BOLD responses in CNS structures where the sensation of pain is mediated (ie, somatosensory cortex). Preclinical studies have demonstrated that antagonist action on $\mathrm{NK}_{1}$ receptors attenuated the behavioral response to noxious stimulation (Xu et al, 1992). Suzuki et al (2003) showed an attenuation of electrophysiological responses to noxious heat in the dorsal horn neurons in $\mathrm{NK}_{1}$ receptor-knockout mice, whereas Budai et al (2007) reported diminished activity of on-cells in rostral ventral medulla following application of an $\mathrm{NK}_{1}$ receptor antagonist (L-733,060). The source of potentation in the supraspinal limbic circuitry observed here could arise from the coupling between antagonist action on $\mathrm{NK}_{1}$ receptors and the 5-HT system (Gobert et al, 2009; Valentino et al, 2003). One main finding by Valentino et al was a characterization of the 5HT-driven inhibition of neuronal activity following substance $\mathrm{P}$ administration: an effect suppressed by treatment with an $\mathrm{NK}_{1}$ antagonist (Sendide). Also, the fact that $\mathrm{NK}_{1}$ receptor as well as substance $\mathrm{P}$ activity may be distinct between spinal (dorsal root horn) and supraspinal limbic structures may need to be taken into consideration. Recent preclinical work involving the dorsal root horn and hippocampus demonstrated that noxious stimulation results in an upregulation of $\mathrm{NK}_{1}$ receptor expression in the dorsal root horn, whereas $\mathrm{NK}_{1}$ receptor expression was downregulated in the hippocampus (Duric and McCarson, 2007). Decreased $\mathrm{NK}_{1}$ receptor expression in limbic structures in chronic pain patients has also been observed (Linnman et al, 2010). Thus, the disparate $\mathrm{NK}_{1}$ receptor properties between spinal and supraspinal regions could be offsetting and prohibit an analgesic effect.

\section{Combining BOLD fMRI and Subjective Pain Ratings during Analgesic Evaluation}

One important aspect of this investigation was a comparison of effect size between BOLD fMRI and subjective pain rating measures. For this comparison, the effect size for BOLD fMRI was consistently greater, particularly for the BUP cohort (Figure 5). Effect size results suggest a more consistent or less variable imaging-based readout in comparison with subjective pain ratings. Given the intrinsic variability of pain ratings, combining this subjective measure with an objective characterization of CNS pain responses may yield a more comprehensive evaluation of analgesic efficacy. Recent works have demonstrated the difficulty in obtaining and interpreting subjective pain reports from trial to trial (Borsook et al, 2011a,b; Lin et al, 2011; Victor et al, 2008). Having an objective and translatable biomarker that links to the sensation and perception of pain could be highly useful in, for example, an early-phase clinical study involving a small, yet sufficiently powered subject population. phMRI and fMRI data can supplement VAS pain ratings, and de-risk such highly variable data, prior to embarking on large-scale clinical trials.

CNS circuits mediating pain drive what an individual experiences perceptually as well as what an individual outputs behaviorally (ie, subjective pain ratings). However, pain ratings are accounts specific to what a human experiences during a painful event, whereas the activity in 
the CNS is likely a more universal measure across mammalian species (eg, rats, non-human primates, and humans), and may yield an objective translatable biomarker for pain. VAS pain ratings alone cannot yield information on the nature of the pain that is experienced: a feature that may be of great importance when characterizing the phenotype of different chronic pain conditions (eg, fibromyalgia, chronic back pain, osteoarthritis, or trigeminal neuralgia) (Woolf, 2010). Such information could be relevant for determining which mechanism of action would be effective for a particular chronic pain disease.

\section{Application to Drug Development}

Typical preclinical and clinical evaluation of potential CNSactive analgesics includes a range of in vitro and in vivo measurements, target engagement (where possible), and behavioral assessments. Nonetheless, even after undergoing such extensive evaluation using these procedures, it is often unknown exactly why a pharmacological compound is effective or not for pain treatment in humans. Determining how a pharmacological compound alters the functionality of the CNS during acute or chronic pain states may provide such indications, or at least provide a signal that can help refine subsequent clinical assessment of the compound. In general, early clinical drug development functional imaging studies in healthy volunteers can provide a potential means to (a) assess the central pharmacodynamic activity of a novel compound and (b) determine the mechanistic engagement by the compound of brain systems linked either to the molecular target or to the target disease state. This would be particularly useful if robust preclinical imaging data could predict with confidence signal changes expected in human subjects. In the case of pain, studies in healthy subjects enable compound activity to be assessed in man with the advantages of a relatively homogeneous sample, ease of recruiting, and the absence of confounding concomitant medications. These conditions are helpful in obtaining a clear, dose-dependent signal that can be of value to inform on dose selection in subsequent trials. However, the degree to which pharmacological effects on relevant brain systems in healthy subjects relate to efficacy in clinical pain conditions remains an open question. The differential findings between the actions of BUP and APREP on functional connectivity and evoked BOLD response in the present study are encouraging in this respect and provide hypotheses to be confirmed if this phMRI/fMRI approach is to be applied prospectively in analgesic drug development. Ongoing and future studies involving both pain imaging and clinical evaluations using additional pharmacological compounds of varying mechanisms known or proposed to have analgesic properties (or not) will help to further address the role of imaging methods in analgesic discovery and development.

In conclusion, phMRI revealed direct effects of both BUP and APREP on the baseline BOLD signal in brain regions consistent with their respective target receptor distributions. However, pharmacological modulation of functional connectivity and attenuation of noxious heat-evoked BOLD responses in the pain-processing pathways were only observed following BUP administration. This investigation demonstrates how CNS-acting drugs can be objectively compared, and how the phMRI/fMRI methodology may be used to complement conventional measures and better evaluate candidate analgesics in small subject cohorts.

\section{ACKNOWLEDGEMENTS}

We thank Dr Brigitte Robertson and Ms Margaret Grant for assistance in preparing this manuscript. This study was funded by the Imaging Consortium for Drug Development.

\section{DISCLOSURE}

Adam J Schwarz, Smriti Iyengar, and David Bleakman are employed by Eli Lilly and Company. Alexandre Coimbra, Richard Baumgartner, Jeffery L Evelhoch, and Richard Hargreaves are employed by Merck and Company. Soujanya Neni, Gary Maier, and Brigitte Robertson are employed by Sunovion Incorporated. David Borsook and Lino Becerra currently receive research grants from Glaxo SmithKline and Merck and Company.

\section{REFERENCES}

Baliki MN, Geha PY, Apkarian AV, Chialvo DR (2008). Beyond feeling: chronic pain hurts the brain, disrupting the defaultmode network dynamics. J Neurosci 28: 1398-1403.

Baliki MN, Geha PY, Fields HL, Apkarian AV (2010). Predicting value of pain and analgesia: nucleus accumbens response to noxious stimuli changes in the presence of chronic pain. Neuron 66: $149-160$.

Becerra L, Breiter HC, Wise R, Gonzalez RG, Borsook D (2001). Reward circuitry activation by noxious thermal stimuli. Neuron 32: 927-946.

Becerra L, Harter K, Gonzalez RG, Borsook D (2006). Functional magnetic resonance imaging measures of the effects of morphine on central nervous system circuitry in opioid-naive healthy volunteers. Anesth Analg 103: 208-216; table of contents.

Benoist JM, Kayser V, Gautron M, Guilbaud G (1983). Low dose of morphine strongly depresses responses of specific nociceptive neurones in the ventrobasal complex of the rat. Pain 15: 333-344.

Bergstrom M, Hargreaves RJ, Burns HD, Goldberg MR, Sciberras D, Reines SA et al (2004). Human positron emission tomography studies of brain neurokinin 1 receptor occupancy by aprepitant. Biol Psychiatry 55: 1007-1012.

Bingel U, Glascher J, Weiller C, Buchel C (2004). Somatotopic representation of nociceptive information in the putamen: an event-related fMRI study. Cereb Cortex 14: 1340-1345.

Borras MC, Becerra L, Ploghaus A, Gostic JM, DaSilva A, Gonzalez RG et al (2004). fMRI measurement of CNS responses to naloxone infusion and subsequent mild noxious thermal stimuli in healthy volunteers. J Neurophysiol 91: 2723-2733.

Borsook D, Becerra L, Hargreaves R (2011a). Biomarkers for chronic pain and analgesia. Part 1: the need, reality, challenges, and solutions. Discov Med 11: 197-207.

Borsook D, Becerra L, Hargreaves R (2011b). Biomarkers for chronic pain and analgesia. Part 2: how, where, and what to look for using functional imaging. Discov Med 11: 209-219.

Budai D, Khasabov SG, Mantyh PW, Simone DA (2007). NK-1 receptors modulate the excitability of ON cells in the rostral ventromedial medulla. J Neurophysiol 97: 1388-1395.

Budd K (1981). High dose buprenorphine for postoperative analgesia. Anaesthesia 36: 900-903. 
Christoph T, Kogel B, Schiene K, Meen M, De Vry J, Friderichs E (2005). Broad analgesic profile of buprenorphine in rodent models of acute and chronic pain. Eur J Pharmacol 507: 87-98.

Coghill RC, Sang CN, Maisog JM, Iadarola MJ (1999). Pain intensity processing within the human brain: a bilateral, distributed mechanism. J Neurophysiol 82: 1934-1943.

Cohen J (1988). Statistical Power Analysis for the Behavioral Sciences 2nd edn. Lawrence Erlbaum Associates: New Jersey.

Cordes D, Haughton VM, Arfanakis K, Carew JD, Turski PA, Moritz $\mathrm{CH}$ et al (2001). Frequencies contributing to functional connectivity in the cerebral cortex in "resting-state" data. AJNR Am J Neuroradiol 22: 1326-1333.

Cowan A, Lewis JW, Macfarlane IR (1977). Agonist and antagonist properties of buprenorphine, a new antinociceptive agent. $\mathrm{Br} J$ Pharmacol 60: 537-545.

Duric V, McCarson KE (2007). Neurokinin-1 (NK-1) receptor and brain-derived neurotrophic factor (BDNF) gene expression is differentially modulated in the rat spinal dorsal horn and hippocampus during inflammatory pain. Mol Pain 3: 32.

Eippert F, Bingel U, Schoell ED, Yacubian J, Klinger R, Lorenz J et al (2009). Activation of the opioidergic descending pain control system underlies placebo analgesia. Neuron 63: 533-543.

Fingelkurts AA, Kivisaari R, Pekkonen E, Ilmoniemi RJ, Kahkonen $S$ (2004). Enhancement of GABA-related signalling is associated with increase of functional connectivity in human cortex. Hum Brain Mapp 22: 27-39.

Fink GR, Frackowiak RS, Pietrzyk U, Passingham RE (1997). Multiple nonprimary motor areas in the human cortex. J Neurophysiol 77: 2164-2174.

Fox MD, Raichle ME (2007). Spontaneous fluctuations in brain activity observed with functional magnetic resonance imaging. Nat Rev Neurosci 8: 700-711.

Gobert A, Brocco M, Dekeyne A, Di Cara B, Bouchez G, Lejeune F et al (2009). Neurokinin1 antagonists potentiate antidepressant properties of serotonin reuptake inhibitors, yet blunt their anxiogenic actions: a neurochemical, electrophysiological, and behavioral characterization. Neuropsychopharmacology 34: 1039-1056.

Goldstein DJ, Offen WW, Klein EG, Phebus LA, Hipskind P, Johnson KW et al (2001). Lanepitant, an NK-1 antagonist, in migraine prevention. Cephalalgia 21: 102-106.

Goldstein DJ, Wang O, Saper JR, Stoltz R, Silberstein SD, Mathew NT (1997). Ineffectiveness of neurokinin-1 antagonist in acute migraine: a crossover study. Cephalalgia 17: 785-790.

Greenwald M, Johanson CE, Bueller J, Chang Y, Moody DE, Kilbourn $\mathrm{M}$ et al (2007). Buprenorphine duration of action: muopioid receptor availability and pharmacokinetic and behavioral indices. Biol Psychiatry 61: 101-110.

Greenwald MK, Johanson CE, Moody DE, Woods JH, Kilbourn MR, Koeppe RA et al (2003). Effects of buprenorphine maintenance dose on mu-opioid receptor availability, plasma concentrations, and antagonist blockade in heroin-dependent volunteers. Neuropsychopharmacology 28: 2000-2009.

Heinricher MM, Morgan MM, Fields HL (1992). Direct and indirect actions of morphine on medullary neurons that modulate nociception. Neuroscience 48: 533-543.

Heinricher MM, Morgan MM, Tortorici V, Fields HL (1994). Disinhibition of off-cells and antinociception produced by an opioid action within the rostral ventromedial medulla. Neuroscience 63: 279-288.

Hewitt DJ, Hargreaves RJ, Curtis SP, Michelson D (2009). Challenges in analgesic drug development. Clin Pharmacol Ther 86: $447-450$.

Hietala J, Nyman MJ, Eskola O, Laakso A, Gronroos T, Oikonen V et al (2005). Visualization and quantification of neurokinin-1 (NK1) receptors in the human brain. Mol Imaging Biol 7: 262-272.

Hill R (2000). NK1 (substance P) receptor antagonists - why are they not analgesic in humans? Trends Pharmacol Sci 21: 244-246.
Iannetti GD, Zambreanu L, Wise RG, Buchanan TJ, Huggins JP, Smart TS et al (2005). Pharmacological modulation of painrelated brain activity during normal and central sensitization states in humans. Proc Natl Acad Sci U S A 102: 18195-18200.

Ide S, Minami M, Satoh M, Uhl GR, Sora I, Ikeda K (2004). Buprenorphine antinociception is abolished, but naloxonesensitive reward is retained, in mu-opioid receptor knockout mice. Neuropsychopharmacology 29: 1656-1663.

Jasinski DR, Pevnick JS, Griffith JD (1978). Human pharmacology and abuse potential of the analgesic buprenorphine: a potential agent for treating narcotic addiction. Arch Gen Psychiatry 35: 501-516.

Katz J, Finnerup NB, Dworkin RH (2008). Clinical trial outcome in neuropathic pain: relationship to study characteristics. Neurology 70: 263-272.

Khalili-Mahani N, van Osch MJ, Baerends E, Soeter RP, de Kam M, Zoethout RW et al (2011). Pseudocontinuous arterial spin labeling reveals dissociable effects of morphine and alcohol on regional cerebral blood flow. J Cereb Blood Flow Metab 31: 1321-1333.

Kogel B, Christoph T, Strassburger W, Friderichs E (2005). Interaction of mu-opioid receptor agonists and antagonists with the analgesic effect of buprenorphine in mice. Eur J Pain 9: 599-611.

LaGraize SC, Borzan J, Peng YB, Fuchs PN (2006). Selective regulation of pain affect following activation of the opioid anterior cingulate cortex system. Exp Neurol 197: 22-30.

Leppa M, Korvenoja A, Carlson S, Timonen P, Martinkauppi S, Ahonen J et al (2006). Acute opioid effects on human brain as revealed by functional magnetic resonance imaging. Neuroimage 31: 661-669.

Lin CP, Kupper AE, Gammaitoni AR, Galer BS, Jensen MP (2011). Frequency of chronic pain descriptors: implications for assessment of pain quality. Eur J Pain 15: 628-633.

Linnman C, Appel L, Furmark T, Soderlund A, Gordh T, Langstrom B et al (2010). Ventromedial prefrontal neurokinin 1 receptor availability is reduced in chronic pain. Pain 149: 64-70.

Lutfy K, Cowan A (2004). Buprenorphine: a unique drug with complex pharmacology. Curr Neuropharmacol 2: 395-402.

Lutfy K, Eitan S, Bryant CD, Yang YC, Saliminejad N, Walwyn W et al (2003). Buprenorphine-induced antinociception is mediated by mu-opioid receptors and compromised by concomitant activation of opioid receptor-like receptors. J Neurosci 23: 10331-10337.

Maihofner C, Baron R, DeCol R, Binder A, Birklein F, Deuschl G et al (2007). The motor system shows adaptive changes in complex regional pain syndrome. Brain 130(Part 10): 2671-2687.

Manning BH, Morgan MJ, Franklin KB (1994). Morphine analgesia in the formalin test: evidence for forebrain and midbrain sites of action. Neuroscience 63: 289-294.

Mansour A, Khachaturian H, Lewis ME, Akil H, Watson SJ (1988). Anatomy of CNS opioid receptors. Trends Neurosci 11: 308-314.

Nakamura A, Yamada T, Goto A, Kato T, Ito K, Abe Y et al (1998). Somatosensory homunculus as drawn by MEG. Neuroimage 7(4 Part 1): 377-386.

Napadow V, LaCount L, Park K, As-Sanie S, Clauw DJ, Harris RE (2010). Intrinsic brain connectivity in fibromyalgia is associated with chronic pain intensity. Arthritis Rheum 62: 2545-2555.

Nicholson AN (1978). Visual analogue scales and drug effects in man. Br J Clin Pharmacol 6: 3-4.

Patel L, Lindley C (2003). Aprepitant-a novel NK1-receptor antagonist. Expert Opin Pharmacother 4: 2279-2296.

Pendse G, Borsook D, Becerra L (2009). Enhanced false discovery rate using Gaussian mixture models for thresholding fMRI statistical maps. Neuroimage 47: 231-261.

Pendse GV, Schwarz AJ, Baumgartner R, Coimbra A, Upadhyay J, Borsook D et al (2010b). Robust, unbiased general linear model estimation of phMRI signal amplitude in the presence of 
variation in the temporal response profile. J Magn Reson Imaging 31: 1445-1457.

Phan KL, Wager T, Taylor SF, Liberzon I (2002). Functional neuroanatomy of emotion: a meta-analysis of emotion activation studies in PET and fMRI. Neuroimage 16: 331-348.

Sante AB, Nobre MJ, Brandao ML (2000). Place aversion induced by blockade of mu or activation of kappa opioid receptors in the dorsal periaqueductal gray matter. Behav Pharmacol 11: 583-589.

Schwarz AJ, Gozzi A, Bifone A (2009). Community structure in networks of functional connectivity: resolving functional organization in the rat brain with pharmacological MRI. Neuroimage 47: 302-311.

Singer T, Seymour B, O'Doherty J, Kaube H, Dolan RJ, Frith CD (2004). Empathy for pain involves the affective but not sensory components of pain. Science 303: 1157-1162.

Smith SM, Nichols TE (2009). Threshold-free cluster enhancement: addressing problems of smoothing, threshold dependence and localisation in cluster inference. Neuroimage 44: 83-98.

Sora I, Elmer G, Funada M, Pieper J, Li XF, Hall FS et al (2001). $\mathrm{Mu}$ opiate receptor gene dose effects on different morphine actions: evidence for differential in vivo mu receptor reserve. Neuropsychopharmacology 25: 41-54.

Steingrimsdottir OA, Vollestad NK, Roe C, Knardahl S (2004). Variation in reporting of pain and other subjective health complaints in a working population and limitations of single sample measurements. Pain 110: 130-139.

Suzuki R, Hunt SP, Dickenson AH (2003). The coding of noxious mechanical and thermal stimuli of deep dorsal horn neurones is attenuated in NK1 knockout mice. Neuropharmacology 45: 1093-1100.

Tershner SA, Helmstetter FJ (2000). Antinociception produced by $\mathrm{mu}$ opioid receptor activation in the amygdala is partly dependent on activation of mu opioid and neurotensin receptors in the ventral periaqueductal gray. Brain Res 865: 17-26.

Tzourio-Mazoyer N, Landeau B, Papathanassiou D, Crivello F, Etard O, Delcroix N et al (2002). Automated anatomical labeling of activations in SPM using a macroscopic anatomical parcellation of the MNI MRI single-subject brain. Neuroimage 15: 273-289.

Upadhyay J, Pendse G, Anderson J, Schwarz AJ, Baumgartner R, Coimbra A et al (2010). Improved characterization of
BOLD responses for evoked sensory stimuli. Neuroimage 49: 2275-2286.

Valentino RJ, Bey V, Pernar L, Commons KG (2003). Substance P acts through local circuits within the rat dorsal raphe nucleus to alter serotonergic neuronal activity. J Neurosci 23: 7155-7159.

Vaughn C, Ingram S, Connor M, Christie M (1997). How opioids inhibit GABA release. Nature 390: 411-414.

Victor TW, Jensen MP, Gammaitoni AR, Gould EM, White RE, Galer BS (2008). The dimensions of pain quality: factor analysis of the Pain Quality Assessment Scale. Clin J Pain 24: 550-555.

Wise RG, Rogers R, Painter D, Bantick S, Ploghaus A, Williams P et al (2002). Combining fMRI with a pharmacokinetic model to determine which brain areas activated by painful stimulation are specifically modulated by remifentanil. Neuroimage 16: 999-1014.

Woolf CJ (2010). Overcoming obstacles to developing new analgesics. Nat Med 16: 1241-1247.

Woolrich MW, Behrens TE, Beckmann CF, Jenkinson M, Smith SM (2004). Multilevel linear modelling for FMRI group analysis using Bayesian inference. Neuroimage 21: 1732-1747.

Xu XJ, Dalsgaard CJ, Wiesenfeld-Hallin Z (1992). Intrathecal CP96,345 blocks reflex facilitation induced in rats by substance $\mathrm{P}$ and C-fiber-conditioning stimulation. Eur J Pharmacol 216: 337-344.

Yaksh TL, Rudy TA (1978). Narcotic analgestics: CNS sites and mechanisms of action as revealed by intracerebral injection techniques. Pain 4: 299-359.

Yang SW, Follett KA, Piper JG, Ness TJ (1998). The effect of morphine on responses of mediodorsal thalamic nuclei and nucleus submedius neurons to colorectal distension in the rat. Brain Res 779: 41-52.

Zubieta JK, Greenwald MK, Lombardi U, Woods JH, Kilbourn MR, Jewett DM et al (2000). Buprenorphine-induced changes in mu-opioid receptor availability in male heroin-dependent volunteers: a preliminary study. Neuropsychopharmacology 23: 326-334.

Zubieta JK, Smith YR, Bueller JA, Xu Y, Kilbourn MR, Jewett DM et al (2001). Regional mu opioid receptor regulation of sensory and affective dimensions of pain. Science 293: 311-315.

Supplementary Information accompanies the paper on the Neuropsychopharmacology website (http://www.nature.com/npp) 